Ann. Sci. forest., 1974, 31 (3), 161-169

\title{
DÉTERMINATION DE L'ANGLE DE LA FIBRE TORSE D'ARBRES SUR PIED \\ A L'AIDE D'UN TRACEUR RADIOACTIF
}

\author{
R. KELLER, P. AZOEUF* et R. HOSLIN* \\ Station de Recherches sur la Qualité des Bois \\ Centre national de Recherches forestières, I.N.R.A., \\ Champenoux, 54280 Seichamps \\ * Section d'Application de la Radioactivité \\ Commissariat à l'Énergie Atomique \\ Saclay, 91190 Gif-sur-Yvette
}

\section{RÉSUMÉ}

Cet article fait le point des premiers essais entrepris pour mesurer l'angle de la fibre torse des arbres sur pied grâce à un traceur radioactif. Ce dernier est injecté dans les accroissements annuels périphériques et transporté par la sève ascendante suivant la direction générale des fibres, impressionne un film appliqué contre l'écorce à une certaine distance au-dessus du point d'injection. La mesure de l'angle se fait facilement sur le film.

Cette méthode ne cause pratiquement pas de dégâts aux arbres et pourrait permettre une étude plus systématique de cette caractéristique, notamment à l'occasion de programmes d'amélioration génétique.

\section{1. - INTRODUCTION}

L'angle de la fibre torse des arbres sur pied est une caractéristique qu'il est utile de prendre en compte dans un programme d'amélioration englobant des critères de qualité $\mathrm{du}$ bois.

En effet, un angle trop élevé confère au bois de mauvaises propriétés technologiques et l'on a intérêt à éviter de produire des arbres à fibre trop torsadée.

Il semble bien (J. Krahl-Urban, 1967, H. Krempl, 1970) que ce phénomène soit sous une dépendance génétique et il est intéressant de le mesurer pour tenter de connaître ses propriétés, en particulier son héritabilité. 
Le but du présent article est de décrire une nouvelle méthode de mesure de l'angle de la fibre torse des arbres sur pied moins dommageable pour eux que celle qui a été utilisée jusqu'à présent.

\section{2. - JUSTIFICATION ET AVANTAGES DE L'UTILISATION D'UN TRACEUR RADIOACTIF}

On pourrait penser que la mesure de l'angle $\alpha$ de la fibre torse d'un arbre sur pied est immédiate quand un angle est apparent sur l'écorce. En fait, cet angle, formé par la disposition relative d'éléments cellulaires extérieurs à l'assise subéro-phellodermique, donc anciens, n'est pas nécessairement égal à celui que l'on cherche à mesurer sur les couches les plus externes, donc les plus récentes, du bois formé par l'assise libéro-ligneuse et situé à l'intérieur de celle-ci (figure 1).

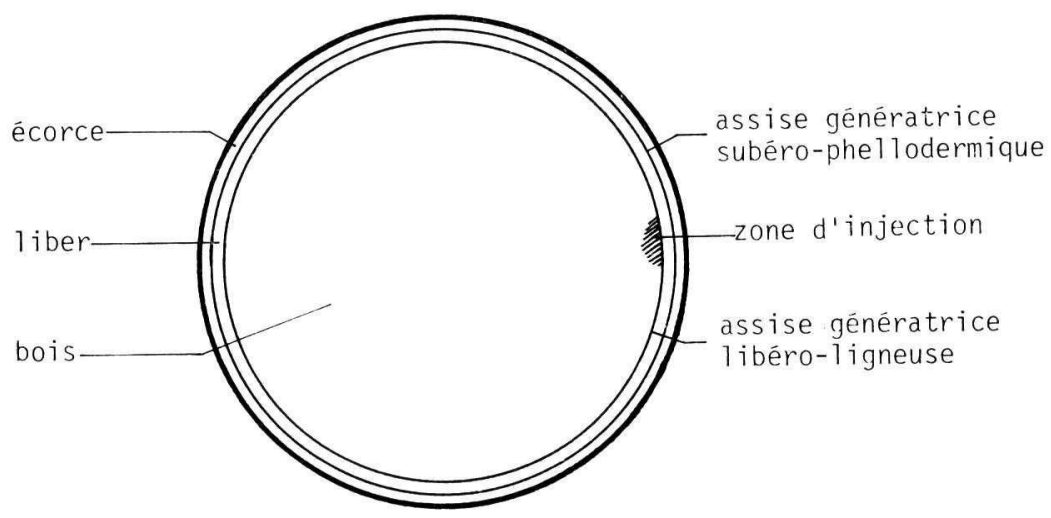

FIG. 1. - Localisation de la zone d'injection

FIG. 1. - Injection zone site

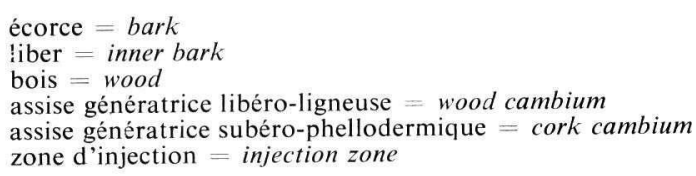

D'autre part, l'écorce peut masquer ce que l'on cherche à mesurer du fait que, chez de nombreuses essences, elle est fissurée et irrégulière.

La méthode mise au point à l'étranger (WheEler R.-T., 1964, Fielding J.-M., 1967) et utilisée jusqu'ici à la Station de Recherches sur la Qualité des Bois, consistait à enlever l'écorce et le liber de l'arbre sur une surface de quelques $\mathrm{cm}^{2}$ (à l'aide d'une scie-cloche ou d'un emporte-pièce) afin de pouvoir appliquer sur le bois, dont on rendait visible l'orientation générale des fibres à l'aide d'une mine de crayon pointue, un rapporteur s'adaptant exactement dans la fenêtre découpée dans l'écorce et le liber. La lecture de l'angle se faisait par rapport à une perpendiculaire à l'axe de l'arbre; cette perpendiculaire était matérialisée par la trace laissée sur les fibres par le tranchant d'un ciseau à bois sur lequel deux 
miroirs formant un dièdre et portant chacun un trait repère (trace de l'intersection avec les deux miroirs d'un plan perpendiculaire à l'arête du dièdre et au tranchant du ciseau) permettaient à l'opérateur de déterminer un plan passant par l'axe général de l'arbre.

Ce procédé, qui a été assez largement utilisé (Keller R., 1973), a l'inconvénient de faire une blessure assez importante à l'arbre (le diamètre de la fenêtre ayant $4 \mathrm{~cm}$ ), et on a cherché à mettre au point une autre méthode présentant moins d'inconvénients.

L'idée d'utiliser un traceur radioactif à cette fin revient à A. FrAnClET, alors à la Station d'Amélioration des Arbres forestiers du C.N.R.F. Si l'on admet que le courant de sève ascendante dans les couches les plus récentes du bois suit de façon préférentielle une succession d'éléments cellulaires ordonnés suivant 1'hélice de la fibre torse, il devrait être possible d'en mesurer le pas en y injectant une solution contenant un corps radioactif qui impressionnerait un film disposé au-dessus du point d'injection sur l'écorce même de l'arbre. Selon P.-W. Owston et al. (1970), des mesures faites sur des arbres (Pinus resinosa et Pinus contorta) à qui l'on avait fait absorber par les racines des solutions de radioisotopes ont montré que, dans la partie inférieure des troncs (jusqu'à environ $5 \mathrm{~m}$ de hauteur), le flux de sève ascendante marquée suit un chemin étroit et spiralé, les radioisotopes restant concentrés à la périphérie du bois, dans les 2 à $3 \mathrm{~cm}$ les plus extérieurs. Ainsi guidés par les éléments anatomiques et séparés du film par relativement peu de bois ou d'écorce, les éléments radioactifs sont capables en un certain temps d'impressionner le film suivant l'hélice de la fibre torse.

Mis à part le trou de sondage préparatoire à l'injection et, éventuellement, un amincissement de l'écorce à faire avant d'appliquer le film contre le tronc, cette méthode ne cause pas de blessure importante à l'arbre. D'autre part, le film étant placé relativement loin du point d'injection, la mesure de l'angle pourra se faire probablement avec plus de précision que lorsqu'on utilise le rapporteur de petites dimensions dont il a été question ci-dessus. Pour ces raisons, des essais ont été tentés qui, bien qu'exploratoires, ont donné les résultats consignés dans ce qui suit.

\section{3. - MATÉRIEL ET MÉTHODE}

\section{1. - Principe de la méthode}

Comme on l'a déjà dit, il consiste à injecter un traceur radioactif dans la sève brute qui le véhicule dans les cellules du bois. Ce traceur impressionne, par émission d'un rayonnement $\beta$, un film sensible placé sur l'écorce à une distance connue du point d'injection, au-dessus de celui-ci.

La mesure de l'angle formé par la trace de la trajectoire du radioélément sur le film et la génératrice du tronc passant par le point d'injection permet de déterminer simplement l'angle de torsion cherché.

\section{2. - Choix du radioisotop?}

Le choix du radioisotope utilisé est orienté par plusieurs critères :

- il doit avoir une période, ou temps au bout duquel son activité diminue de moitié, relativement longue pour permettre l'impression du film radiographique, et cela en fonction de la distance entre le point d'injection et l'emplacement du film d'une part, et de la vitesse du déplacement de la sève d'autre part,

- il doit avoir une période relativement courte pour diminuer les risques d'irradiation et de contamination, 
— pour impressionner avec un rendement suffisant le film, il doit être au moins un émetteur $\beta$ et l'énergie de son rayonnement $\beta$ doit être assez grande pour traverser l'écorce, et cela d'autant plus que la distance entre les cellules où circule la sève et le film est élevée,

- la solution injectée doit être physiologiquement compatible avec la sève pour, par exemple, ne pas précipiter au point d'injection.

Pour ces différentes raisons, notre choix s'est porté sur le potassium $42\left({ }^{42} \mathrm{~K}\right)$ dont les caractéristiques sont les suivantes:

$$
\begin{aligned}
\text { — période } \mathrm{T}: & 12,4 \mathrm{heures} \\
\text { — énergie } \beta & : 3,52 \mathrm{Mev}(82 \%) \\
& 2,03 \mathrm{Mev}(18 \%) \\
\text { — énergie } \gamma: & : 1,52 \mathrm{Mev}(18 \%)^{*}
\end{aligned}
$$

Il est obtenu en pile par réaction $(n, \gamma)$ à partir de l'isotope naturel ${ }^{41} \mathbf{K}$ dont l'abondance isotopique est de $6,88 \%$ et la section efficace de 1,30 barns.

Il est livré par le C.E.A. de Saclay sous forme de $\mathrm{KCl}$ d'activité spécifique comprise entre 0,04 et $0,16 \mathrm{mci} / \mathrm{mg}$. Son activité volumique est de 0,5 à $2 \mathrm{mci} / \mathrm{ml}$.

\section{3. - Technique expérimentale}

\section{Injection.}

En assimilant le tronc de l'arbre à étudier à un cylindre, on y matérialise une génératrice. En un point de celle-ci, on perce, à l'aide d'un foret, un trou de $3 \mathrm{~mm}$ de diamètre et d'une profondeur telle qu'il atteigne le bois, après avoir traversé l'assise génératrice libéro-ligneuse. A l'aide d'une seringue et d'une aiguille hypodermique, on introduit dans ce trou un certain volume de solution de ${ }^{42} \mathbf{K}$, en général entre 0,1 et $0,3 \mathrm{ml}$, cela pour une activité comprise entre $0,1 \mathrm{mci}$ et $1 \mathrm{mci}$. Une activité de 0,1 à 0,2 mci doit être suffisante.

\section{Autoradiographie.}

A une distance en général de $30 \mathrm{~cm}$ au-dessus du point d'injection, on place un film sensible « double face » du type Kodirex de dimensions $30 \mathrm{~cm} \times 40 \mathrm{~cm}$ (le bord inférieur du film à $30 \mathrm{~cm}$ du point d'injection, le bord supérieur à $60 \mathrm{~cm}$ ).

Sa durée d'exposition est essentiellement fonction de la vitesse de déplacement de la sève car il faut évidemment un certain temps pour que ${ }^{42} \mathrm{~K}$ parcoure la distance séparant le point d'injection du bord supérieur du film.

Comme l'indique le tableau récapitulatif des essais (Tabl. 1), ce temps d'exposition peut varier de 1 heure à 48 heures; il dépend de l'essence étudiée et de la période dans la saison de végétation. On a intérêt à opérer au moment où la circulation de la sève est active, ce qui dépend des conditions de milieu et de climat. En particulier, il est probable que les essais $N^{\text {os }} 10,11,14,20 \mathrm{a}, 20 \mathrm{~b}$ et 21 ont été trop tardifs pour des arbres poussant dans la région parisienne.

La distance de $30 \mathrm{~cm}$ est un compromis qui tient compte de ce que le chemin à parcourir par ${ }^{4} \mathrm{~K}$ doit être assez petit pour qu'il ne perde pas trop d'activité et assez grand pour que la précision dans la détermination de l'angle cherché soit suffisante (voir tabl. 1).

\section{4. - Détermination de l'angle de la fibre torse}

L'angle $\alpha$ de la fibre torse est donné par la relation :

$$
\operatorname{tg} \alpha=\frac{\mathrm{AB}}{\mathrm{OA}} \text { (figure 2) }
$$

où $\mathrm{AB}$ est la longueur de l'arc de cercle $\mathrm{AB}$

OA la distance du bord supérieur du film au point d'injection mesurée suivant la génératrice définie ci-dessus.

\footnotetext{
* Énergie et pourcentage relatifs au rayonnement $\gamma$ le plus important.
} 
TABLEAU 1 - TABLE 1

Tableau récapitulant quelques essais au ${ }^{42} \mathrm{~K}$

Table showing the conditions of the trials with ${ }^{42} \mathrm{~K}$

\begin{tabular}{|c|c|c|c|c|c|c|c|c|c|c|}
\hline $\begin{array}{c}\text { Essai } \\
\mathrm{n}\end{array}$ & Date & Espèce & $\begin{array}{l}\text { Diamètre } \\
\text { du tronc } \\
(\mathrm{cm})\end{array}$ & $\begin{array}{l}\text { Épaisseur } \\
\text { d'écorce } \\
\text { (cm) }\end{array}$ & $\begin{array}{l}\text { Volume } \\
\text { injecté } \\
(\mathrm{ml})\end{array}$ & $\begin{array}{c}\text { Activité } \\
\text { injectée } \\
\text { (mci) }\end{array}$ & $\begin{array}{c}\text { Durée de } \\
\text { l'exposition }\end{array}$ & $\begin{array}{l}\text { Distance de la } \\
\text { base du film par } \\
\text { rapport au point } \\
\text { d'injection (cm) }\end{array}$ & Temps & $\begin{array}{l}\text { Vitesse approxi- } \\
\text { mative de la } \\
\text { montée de sève } \\
(\mathrm{cm} / \mathrm{h})\end{array}$ \\
\hline $\begin{array}{l}1 \mathrm{x} \\
2 \\
3\end{array}$ & $\begin{array}{c}15 / 5 / 1973 \\
" \\
17 / 5 / 1973\end{array}$ & $\begin{array}{c}\text { Betula sp. } \\
" ” \\
\text { Abies } \\
\text { grandis }\end{array}$ & $\begin{array}{l}20 \\
25 \\
25\end{array}$ & $\begin{array}{c}0,5 \\
0,5-1 \\
0,5-1\end{array}$ & $\begin{array}{l}0,1 \\
0,1 \\
0,2\end{array}$ & $\begin{array}{l}0,55 \\
0,55 \\
0,10\end{array}$ & $\begin{aligned} 1 \mathrm{~h} 30 \\
2 \mathrm{~h} 15 \\
24 \mathrm{~h}\end{aligned}$ & $\begin{array}{l}30 \\
30 \\
25\end{array}$ & $\begin{array}{c}\text { beau } \\
» \\
\text { couvert }\end{array}$ & $\begin{array}{r}100 \\
18 \\
20\end{array}$ \\
\hline $\begin{array}{c}7 \mathrm{x} \\
10 \mathrm{x} \\
11 \\
14\end{array}$ & $\begin{array}{c}22 / 5 / 1973 \\
29 / 5 / 1973 \\
» \\
25 / 7 / 1973\end{array}$ & $\begin{array}{l}\gg \\
» \\
" \\
\text { Pinus } \\
\text { silvestris }\end{array}$ & $\begin{array}{l}25 \\
18 \\
18 \\
60\end{array}$ & $\begin{array}{l}0,5-1 \\
0,5-1 \\
0,5-1 \\
6\end{array}$ & $\begin{array}{l}0,1 \\
0,5 \\
0,5 \\
0,2\end{array}$ & $\begin{array}{l}0,70 \\
1,70 \\
1,10 \\
0,40\end{array}$ & $\begin{array}{r}4 \mathrm{~h} \\
6 \mathrm{~h} \\
16 \mathrm{~h} \\
24 \mathrm{~h}\end{array}$ & $\begin{array}{l}30 \\
30 \\
30 \\
30\end{array}$ & $\begin{array}{l}\text { beau } \\
\text { couvert } \\
\text { pluie } \\
\text { couvert }\end{array}$ & $\frac{\overline{20}}{3}$ \\
\hline $\begin{array}{l}20 \mathrm{a} \\
20 \mathrm{bx} \\
21 \mathrm{x}\end{array}$ & $\begin{array}{c}21 / 8 / 1973 \\
" \\
"\end{array}$ & $\begin{array}{l}" \prime \\
" \\
\text { Pinus } \\
\text { nigra } \\
\text { (laricio) }\end{array}$ & $\begin{array}{l}50 \\
50 \\
56\end{array}$ & $\begin{array}{l}5 \\
5 \\
7\end{array}$ & $\begin{array}{l}0,3 \\
0,3 \\
0,3\end{array}$ & $\begin{array}{l}1,00 \\
1,00 \\
1,00\end{array}$ & $\begin{array}{l}48 \mathrm{~h} \\
48 \mathrm{~h} \\
48 \mathrm{~h}\end{array}$ & $\begin{array}{r}30 \\
100 \\
30\end{array}$ & $\begin{array}{c}\text { beau } \\
\text { ” } \\
»\end{array}$ & $\begin{array}{l}10 \\
10 \\
10\end{array}$ \\
\hline
\end{tabular}

Les numéros suivis de $\mathrm{x}$ correspondent aux essais qui ont donné les traces les plus nettes sur les films.

The marked with $x$ numbers correspond with the trials which gave the better traces on the films.

essai $=$ trial - date $=$ date - espèce $=$ species - diamètre du tronc $=$ diameter of the trunk at $1,3 \mathrm{~m}$.

épaisseur d'écorce $=$ depth of the bark - volume injecté $=$ injected volume - activité injectée $=$ injected activity:

durée de l'exposition = length of the exposure - distance de la base du film par rapport au point d'injection = range from the point of injection to the base of the film;

temps $=$ weather - beau $=$ sunny - couvert $=$ cloudy - pluie $=$ rainy

vitesse approximative de la monté de sève : approximate speed of the ascending sap ; 


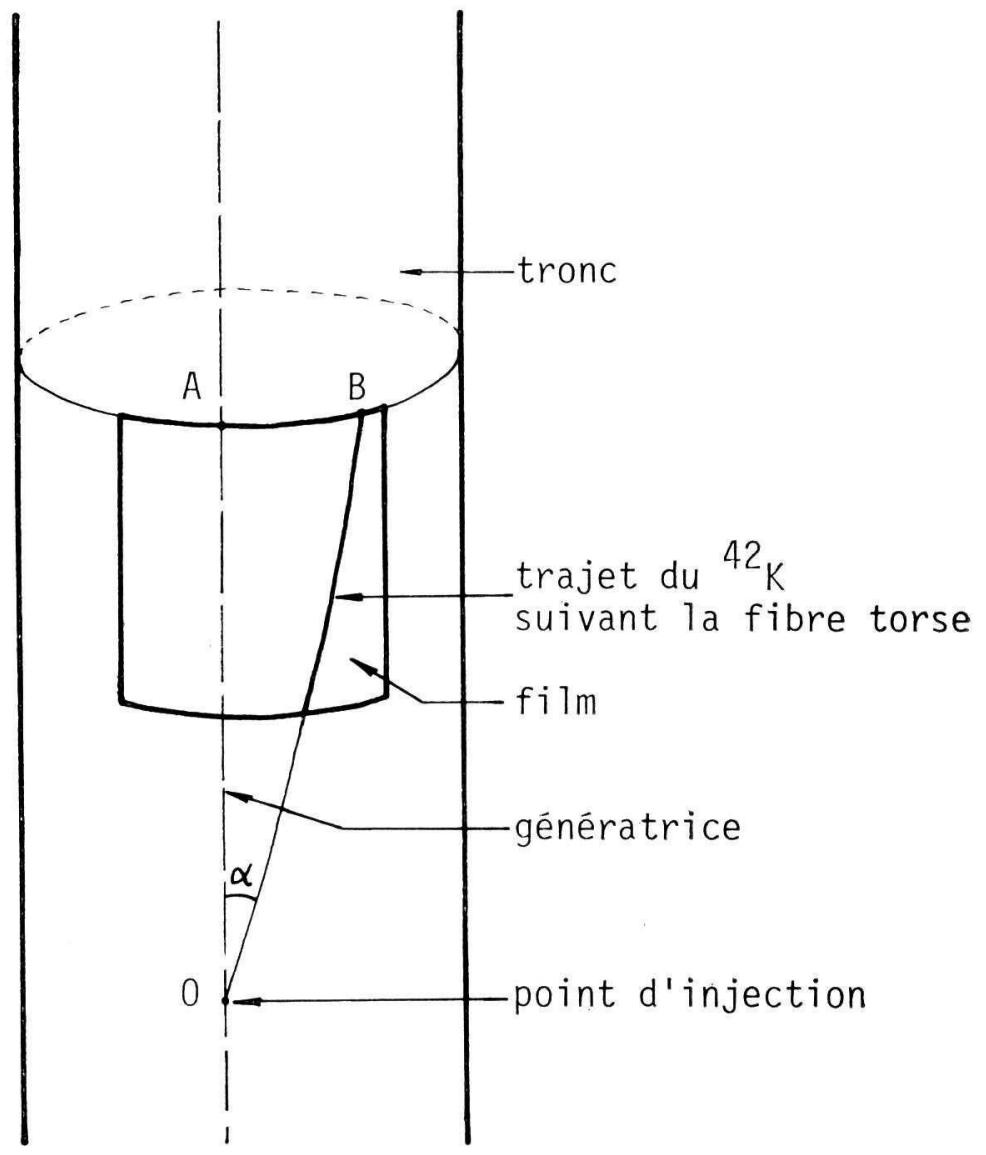

Fig. 2. - Détermination de l'angle de la fibre torse

FIG. 2. - Determination of spiral grain

trajet $\mathrm{du}{ }^{42} \mathrm{~K}$ suivant la fibre torse $={ }^{42} \mathrm{~K}$ path follows the direction of spiral grain.

film $=$ film

génératrice = generating line

tronc $=$ trunk

point d'injection $=$ injection point

Le film étant développé suivant une surface plane, il suffit d'y mesurer la distance AB entre la trace matérialisée de la génératrice et celle laissée par ${ }^{42} \mathrm{~K}$ (figure 3 ).

L'angle $\alpha$ étant généralement faible, on peut se contenter de l'approximation

$$
\alpha_{\mathrm{rad}}=\frac{\mathrm{AB}}{\mathrm{OA}}
$$

A titre d'exemple, on a obtenu, dans le cas du cliché $\mathrm{n}^{\circ} 20 \mathrm{~b}$ de la figure 3 , sur un pin sylvestre :

$\mathrm{AB}=-7,3 \mathrm{~cm}$ (pas de l'hélice à gauche pour

un observateur extérieur)

ce qui conduit à :

$$
\mathrm{OA}=129,7 \mathrm{~cm} \text {, }
$$

$$
\alpha=\frac{-7,3}{129,7} \cdot \frac{400}{6,28}=-3,6 \text { grades. }
$$



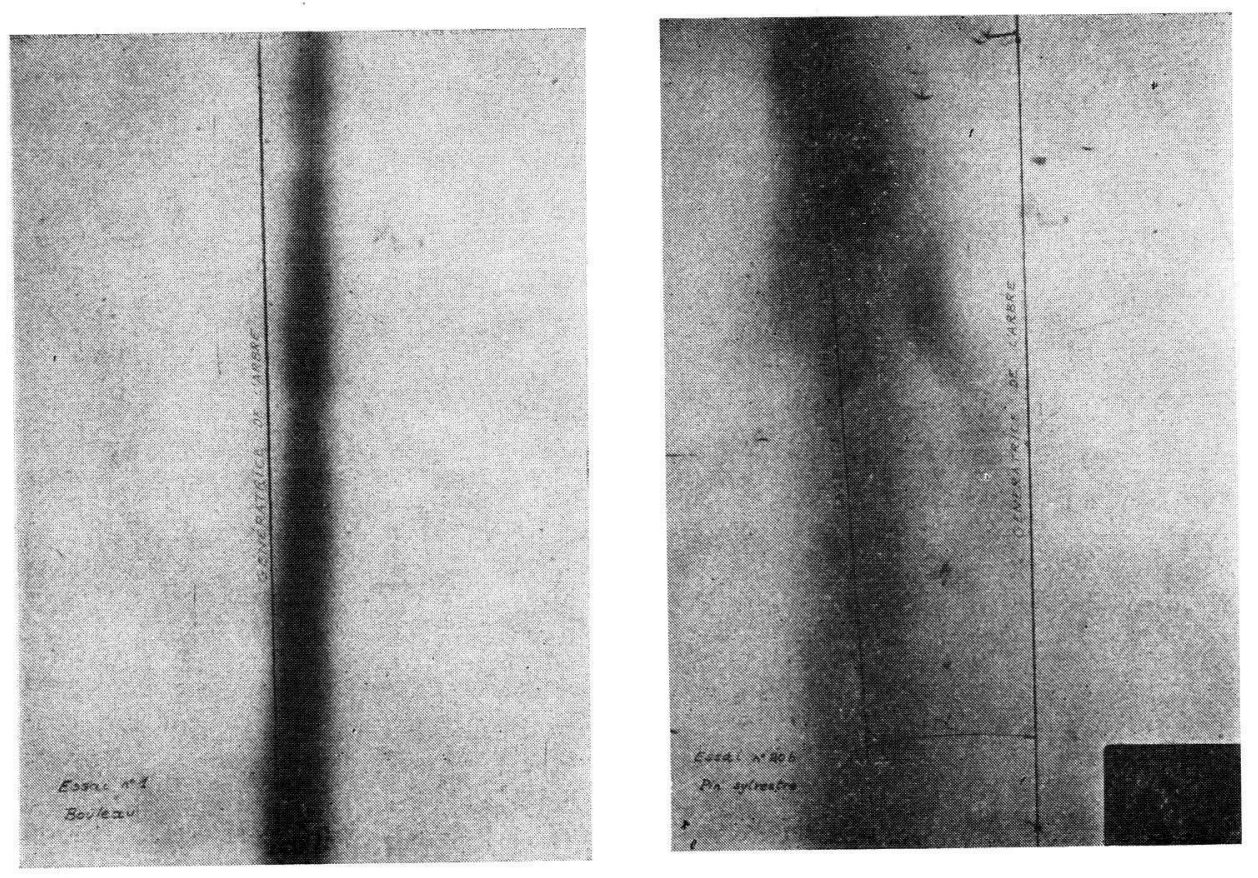

Fig. 3. - Positifs des films obtenus sur bouleau (essai $n^{0} 1$ ) et sur pin sylvestre (essai $n^{\circ} 20 b$ ) - échelle 1/3.

FIG. 3. - Positive prints of films obtained on birch (experiment $n^{0} 1$ )

and scots pine (experiment $n^{\circ} 20 b$ ) - scale 1/3

génératrice de l'arbre = generating line of the trunk

trace de la fibre torse $=$ trace of the spiral grain 
Des mesures faites sur une rondelle de cet arbre, que nous avons ensuite abattu (cernes découpés un à un au ciseau à bois, angle $\alpha$ mesuré au rapporteur par rapport à l'axe de l'arbre), ont donné les résultats suivants :

\begin{tabular}{l|r}
\hline \hline Année & $\alpha$ (grades) \\
& \\
\hline 1973 & -7 \\
1972 & -6 \\
1971 & -5 \\
1970 & -4 \\
1969 & -3 \\
1968 & -8 \\
1967 & +4 \\
1966 & +8 \\
1965 & +5 \\
1964 & +1 \\
1954 & -6 \\
1944 & -6 \\
1934 & -3 \\
1924 & -5 \\
1914 & -2 \\
1908 (moelle) & 0 \\
\hline
\end{tabular}

Si l'on considère les cinq dernières années, cela donne un angle moyen de -5 grades.

\section{4. - CONCLUSION}

L'apparition d'angles positifs dans une longue série d'angles négatifs et la forte variation de l'angle de 1967 à 1968 laissent présager des difficultés dans l'étude du contrôle génétique du caractère " fibre torse »; on se serait attendu en effet à une plus forte stabilité, surtout dans cette zone périphérique d'un arbre âgé, mais un exemple unique ne permet, ni de conclure sur la valeur comparée des résultats des deux procédés, ni d'étudier une éventuelle stabilisation dans l'évolution des angles de la fibre torse.

La méthode utilisant un radioisotope étant non destructive et peu dommageable envers les arbres semble être bien préférable aux deux autres évoquées dans cette note qui obligeaient, soit à abattre l'arbre, soit à lui faire des blessures importantes, et empêchaient pratiquement de prendre en compte cette caractéristique importante de qualité du bois.

En 1974 va être étudiée, à l'occasion d'une campagne de mesures sur de nombreux arbres qui permettra de mieux connaître les limites de la méthode, la possibilité d'utiliser un générateur radioactif qui permettrait au manipulateur de se libérer de l'astreinte d'un approvisionnement fréquent en radioisotope à courte période.

Son principe est le suivant :

On fixe sur une colonne d'échange d'ions un élément radioactif appelé «père » de période longue, de l'ordre du mois ou plus, par exemple. Par décroissance radioactive, ce « père » donne un autre radioélément, appelé « fils », de période plus courte, soit quelques heures. La production du « fils » est continue à partir du « père ». Avec un éluant approprié, on n'extrait de la colonne et d'une manière spécifique que le « fils».

L'intérêt de ce système est de procurer aux équipes de mesures une plus grande autonomie sur le terrain. 


\title{
SUMMARY
}

\author{
TRACER METHOD FOR THE MEASUREMENT OF SPIRAL \\ GRAIN ON STANDING TREES
}

This work summarizes the results of experiments on the application methods to the measurement of spiral grain on standing trees. The marker is injected into the external growth rings and carried by the ascending sap in the direction of the spiral grain; it prints a film which is applied on the bark at a certain distance above the injection point. The measurement of the spiral grain on the film is easy. This method does not cause any great damage to the trees and might permit a more systematic investigation of this characteristic, particularly in the case of a genetic improvement program.

\section{ZUSAMMENFASSUNG}

\section{DIE BESTIMMUNG DES DREHWUCHSWINKELS AN LEBENDEN BÄUMEN NACH MARKIERUNG MIT RADIOISOTOPEN}

Die vorliegende Abhandlung fasst die Ergebnisse von ersten Versuchen zur Bestimmung des Drehwuchswinkels nach Markierung mit Radioisotopen an lebenden Bäumen zusammen.

Das Radioisotop $\left({ }^{42} \mathrm{~K}\right)$ wird in die äusseren Jahrringe injekziert und folgt im aufsteigenden Saftstrom der allgemeinen Faserrichtung. Ein Film wird in einem bestimmten Abstand oberhalb der Injektionstelle an die Rinde angelegt. Der Richtungswinkel der Fasern kann anschliessend leicht am Film gemessen werden.

Diese Methode verursacht praktisch keine Schäden an den Bäumen und dürfte sich für systematische Untersuchungen dieses Merkmals, insbesonders bei forstgenetischen Programmen, gut eignen.

\section{RÉFÉRENCES BIBLIOGRAPHIQUES}

FiELdiNG J. M., 1967. - Spiral grain in Pinus radiata plantations in the Australian Capital territory. Forest Research Institute, Department of National Development Forestry and Timber Bureau, Canberra, Leaflet $\mathrm{n}^{\circ} 103$.

Keller R., 1973. - Caractéristiques du bois de pin maritime, variabilité et transmission héréditaire. Ann. Sci. Forest., XXX, 1, 31-62.

Krahl-Urban J., 1967. - Uber den Drehwuchs bei Buchen. XIV e Congrès de l'IUFRO, exposés t. IX, Groupe de travail entre sections, 22/41, 384-397, Münich.

Krempl H., 1970. - Untersuchungen über den Drehwuchs bei Fichte. Mitteilungen der forstlichen Bundes Versuchanstalt, Wien, fascicule 89.

Owston P.-W., Smith J.-L., Halverson H.-G., 1970. — Development of some radioisotope procedures for measuring water movement in trees. Isotopes and radiation technology, vol. $7, \mathrm{n}^{\circ} 4,396-401$.

WHEELER R.-T., 1964. - Equipement suitable for measuring angle of grain and for obtaining small timber samples from living trees. Commonwealth Forestry Review, vol. 43 (4), no 118. 